\title{
Scanning Tunneling Microscopy Studies of Temperature-Dependent Etching of Diamond (100) by Atomic Hydrogen
}

\author{
R. E. Stallcup II and J. M. Perez \\ Department of Physics, University of North Texas, Denton, Texas 76201
}

(Received 24 October 2000)

\begin{abstract}
We present a technique for obtaining atomic resolution ultrahigh vacuum scanning tunneling microscopy images of diamond (100) films, and use this technique to study the temperature dependence of the etching of epitaxial diamond (100) films by atomic hydrogen. We find that etching by atomic hydrogen is highly temperature dependent, resulting in a rough and pitted surface at $T \approx 200$ and $500{ }^{\circ} \mathrm{C}$, respectively. At $T \approx 1000^{\circ} \mathrm{C}$ etching results in a smooth surface and is highly anisotropic, occurring predominantly in the direction of dimer rows. This observation supports recent theoretical models that propose anisotropic etching as the mechanism for the growth of smooth diamond (100) films.
\end{abstract}

PACS numbers: 81.65.Cf, 68.37.Ef

Diamond films grown using chemical vapor deposition (CVD) [1] have recently attracted considerable interest due to the unique mechanical and electronic properties of this material [2]. CVD growth of diamond films is not as well understood as CVD growth of Si and other semiconductors. Growth of diamond films requires a large ratio of molecular hydrogen $\left(\mathrm{H}_{2}\right)$ to hydrocarbon gas. During growth, $\mathrm{H}_{2}$ is dissociated into atomic hydrogen by a hot-tungsten filament or microwaves. It is known that atomic hydrogen etches away graphite, and promotes diamond growth by terminating the diamond surface in an $s p^{3}$ configuration [1-3]. It is also known that atomic hydrogen etches diamond [1-3]. However, the effects of atomic hydrogen etching of diamond during growth are not well understood. Scanning tunneling microscopy (STM) in ultrahigh vacuum (UHV) has been extensively used to study the etching and growth of $\mathrm{Si}[4,5]$ and other semiconductors [5] at the atomic scale. To our knowledge, UHV STM has not been extensively used to study diamond. Most STM studies of diamond have been performed in air where sample preparation is limited to room temperature techniques and usually a hydrogen-terminated surface [6-8]. A recent study reported that STM was not possible in UHV because the diamond surface was not conductive enough until it was exposed to air [7]. One would like to see results of UHV STM of diamond comparable in quality to those that have been reported for Si.

Epitaxial diamond (100) films are of particular interest because such films grow smooth at substrate temperatures $T \approx 1000{ }^{\circ} \mathrm{C}$ [9], unlike epitaxial diamond (110) and (111) films that grow rough. The growth of smooth diamond (100) films is not well understood. Surface diffusion of adsorbates, which is responsible for the growth of most smooth films, is not widely accepted as responsible for the growth of smooth diamond (100) films because the hydrogen-terminated surface prevents diffusion [3]. Diffusion on the diamond (100) surface during growth has been modeled as occurring along surface sites where hydrogen has been removed by abstraction [10]. However, recent theoretical models have shown that the addition of surface diffusion to models would lead to higher growth rates than are experimentally observed [11]. Other mechanisms that have been proposed to explain the growth of smooth diamond (100) films are anisotropic etching [12] and preferential etching of under-coordinated atoms [11]; however, there has not yet been any direct evidence for these processes. In this paper, we report, for the first time, atomic resolution UHV STM studies of the temperature dependence of the etching of epitaxial diamond (100) films by atomic hydrogen, and these results support the anisotropic etching model.

The epitaxial films were grown on single-crystal diamond substrates that were polished to within $1^{\circ}$ of the (100) direction. The films were grown for two hours in a UHV compatible hot-tungsten filament CVD reactor and were approximately $2 \mu \mathrm{m}$ thick. The reactor is coupled to a UHV STM chamber at a pressure $<10^{-10}$ Torr via an all-metal through valve. The CVD growth pressure was 30 Torr with $\mathrm{H}_{2}$ and methane flow rates of 200 and 0.5 SCCM, respectively. (SCCM denotes cubic centimeter per minute at STP.) In order to increase the conductivity of the films for STM studies, the films were doped with boron during growth by using diborane gas at a concentration of $10 \mathrm{ppm}$ relative to $\mathrm{H}_{2}$. The tungsten filament and substrate temperatures were 2200 and $900{ }^{\circ} \mathrm{C}$, respectively.

After growth, the CVD reactor was evacuated to $1 \times$ $10^{-8}$ Torr and the diamond films were transferred to the UHV STM chamber via a linear translator without contaminating them by exposure to air. After verifying by STM that the films were of good quality, the films were returned to the CVD reactor, via the linear translator, for additional exposure to atomic hydrogen. The $\mathrm{H}_{2}$ flow rate, pressure, and filament temperature were 200 SCCM, 30 Torr, and $2200{ }^{\circ} \mathrm{C}$, respectively. These parameters were the same as those for CVD diamond growth to ensure that the diamond surface was exposed to the same amount of atomic hydrogen flux as during growth. The experiments consisted of exposing the surface to atomic hydrogen at 
substrate temperatures of 200,500 , and $1000{ }^{\circ} \mathrm{C}$. The substrate temperature was measured by monitoring the shift in the $1332 \mathrm{~cm}^{-1}$ Raman peak with temperature [13]. After each exposure, the film was translated to the UHV STM to determine the effect of exposure on the surface structure. Tunneling currents of $1 \mathrm{nA}$ with a sample bias of 1 to $1.5 \mathrm{~V}$ were used.

We found that atomic resolution UHV STM imaging of diamond films after growth was limited to small area scans due to tunneling instabilities that we believe result from physisorbed hydrogen and carbon on the film surface. Tunneling current instabilities caused by this contamination resulted in tip crashes that often rendered the tip useless. The results presented in this paper are without these problems because of three important factors [14]. The first factor is that the films are etched by atomic hydrogen after growth for at least $5 \mathrm{~min}$, thereby removing the excess and loosely bound carbon from the surface. The second factor is that we found that desorbing the loosely bound hydrogen from the surface by heating the substrate to $800{ }^{\circ} \mathrm{C}$ for 1 min results in improvement of the tunneling current stability, which produces clearer STM images. Further heating to $1000{ }^{\circ} \mathrm{C}$ removes the hydrogen-terminated surface and further enhances the STM image quality. These techniques for cleaning the diamond surface can be performed only in UHV. The third factor is the use of ultrasharp tungsten tips [15]. Using these techniques, we obtained atomic resolution images of areas measuring $40 \times 40 \mathrm{~nm}^{2}$ that are comparable in quality to those reported for $\mathrm{Si}$. From these images, the large-scale morphology of the surface was compared with theoretical models.

Figures 1(a) and 1(b) show UHV STM images of an epitaxial diamond (100) film grown as described above after exposure to atomic hydrogen for 5 and $10 \mathrm{~min}$, respectively, at a substrate temperature of $500{ }^{\circ} \mathrm{C}$. A $2 \times 1$ surface reconstruction is observed. The $2 \times 1$ surface after exposure to atomic hydrogen is monohydride terminated [16], and is denoted as diamond (100)- $(2 \times 1): \mathrm{H}$. Two different types of steps are observed, marked $S_{A}$ and $S_{B}$ in Fig. 1(a). $S_{A}$ steps have dimer rows parallel to the step edge, and $S_{B}$ steps have dimer rows perpendicular to the

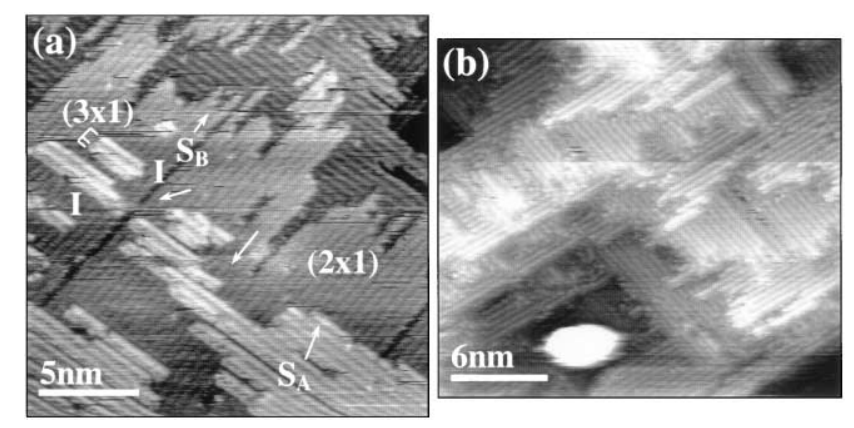

FIG. 1. (a) Hydrogen-terminated diamond (100)- $(2 \times 1): \mathrm{H}$ surface after exposure to atomic hydrogen for $5 \mathrm{~min}$ at $500^{\circ} \mathrm{C}$. (b) Diamond $(100)-(2 \times 1): \mathrm{H}$ surface after exposure to atomic hydrogen for $10 \mathrm{~min}$ at $500{ }^{\circ} \mathrm{C}$ showing an etch pit containing a bright circular structure. step edge. In Fig. 1(a), unmarked arrows indicate regions where $S_{A}$ steps were etched perpendicular to the rows resulting in islands marked I. The islands have a $3 \times 1$ reconstruction consisting of a monohydride-terminated row and a dihydride-terminated row. Local $3 \times 1$ reconstructions on the diamond $(100)-(2 \times 1): H$ surface have been previously reported along step edges [8]. Figure 1(b) shows that the film has square etch pits several atomic layers deep. Each pit was observed to contain a bright circular structure, as shown in Fig. 1(b). We measured a pit density of $1 \times 10^{11} \mathrm{~cm}^{2}$ and conjecture that the pits are due to dislocations. Recent atomic force microscopy studies of diamond (100) films etched by an atomic hydrogen plasma show similar square pits having a distribution that corresponds to that of dislocations [17]. We conjecture that the bright structures in the pits are groups of hydrogen atoms or etch by-products because the structures disappear after the sample is heated to $1000{ }^{\circ} \mathrm{C}$. As shown in Fig. 1(b), the pits are pyramidal in shape and have (111) faces showing that, at $500{ }^{\circ} \mathrm{C}$, the etch rate in the (111) direction is slower than that in the (100) and (110) directions. Etching for an additional $30 \mathrm{~min}$ widened the pits resulting in overlapping of the pits and (111) faceting of the surface [14]. Larger area atomic resolution STM images of the hydrogen-terminated surface were difficult to obtain due to current instabilities, as discussed above.

Using low-energy-electron diffraction (LEED) and electron-energy-loss spectroscopy, it has been reported that heating of the hydrogen-terminated diamond (100)$(2 \times 1)$ : $\mathrm{H}$ surface at $1000^{\circ} \mathrm{C}$ in UHV results in desorption of hydrogen and a nonhydrogen terminated diamond $(100)-(2 \times 1)$ surface [18]. Figures $2-5$ show UHV STM images of the diamond $(100)-(2 \times 1)$ surface after exposure to atomic hydrogen for $5 \mathrm{~min}$ at substrate temperatures of 200,500 , and $1000{ }^{\circ} \mathrm{C}$. In Figs. 2-5, the diamond (100) surface was heated to $1000^{\circ} \mathrm{C}$ for $1 \mathrm{~min}$ in UHV after the atomic hydrogen exposure in order to desorb the hydrogen physisorbed and chemisorbed on the surface. During heating, only $\mathrm{H}_{2}$ was observed using a residual gas analyzer. We find that the stability of the tunneling current increases after the hydrogen desorption. For example, Figs. 3 and 4 show tiled STM images obtained one after the other showing excellent reproducibility.

Figures 2-5 show that the temperature of the surface during exposure to atomic hydrogen has a significant effect on the resulting morphology. We found that the morphologies shown in Figs. 2-5 are due to the atomic hydrogen exposure instead of the subsequent heating at $1000^{\circ} \mathrm{C}$ in UHV. For example, additional heating of the surface at $1000{ }^{\circ} \mathrm{C}$ for $15 \mathrm{~min}$ did not result in any observable changes in morphology. This is consistent with the expected low surface mobility of atoms at $1000{ }^{\circ} \mathrm{C}$ due to the temperature being well below the Debye temperature of diamond of $15900^{\circ} \mathrm{C}$ [3]. In addition, films with any one of the three morphologies shown in Figs. 2-5 could be converted to any of the two other morphologies by 


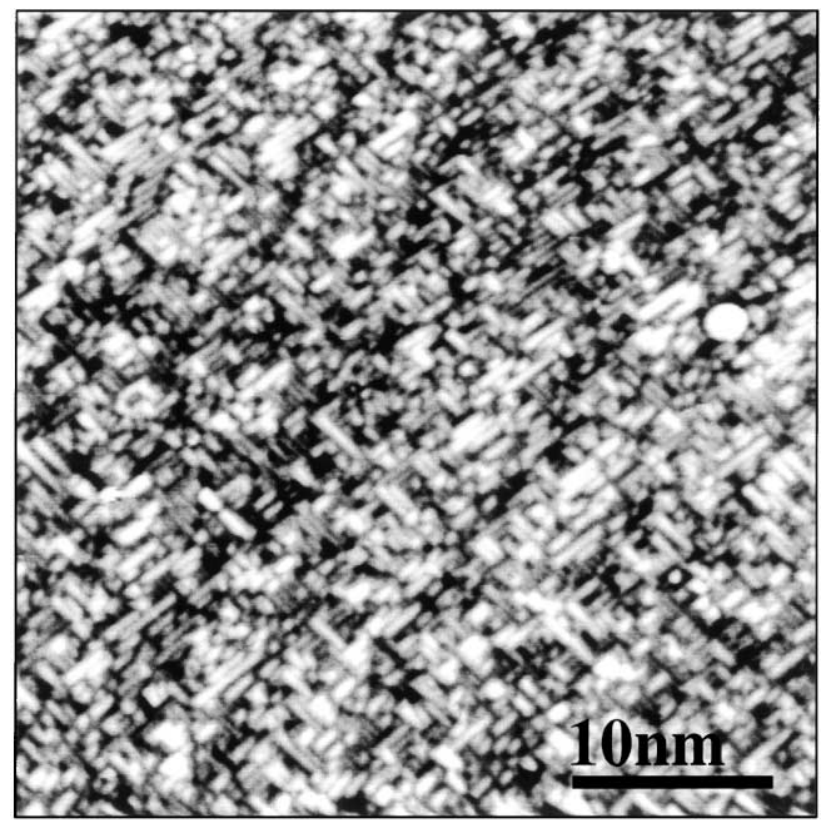

FIG. 2. Nonhydrogen terminated diamond $(100)-(2 \times 1)$ surface after atomic hydrogen exposure for $5 \mathrm{~min}$ at $200{ }^{\circ} \mathrm{C}$.

simply etching at that specific temperature. For example, a film with the smooth morphology shown in Fig. 5 was etched with atomic hydrogen at $200{ }^{\circ} \mathrm{C}$ and converted to a film with a rough morphology such as that shown in Fig. 2. This rough morphology was then changed back to the smooth morphology by etching at $1000{ }^{\circ} \mathrm{C}$.

As shown in Fig. 2, exposure at $200{ }^{\circ} \mathrm{C}$ results in a rough surface at the atomic scale. The largest $2 \times 1$ domain size is about $6 \mathrm{~nm}^{2}$, and there is no clear step direction. A rough surface results when etching is isotropic in which the probability of removal of a surface atom is independent of its coordination [4]. As shown in Fig. 3, exposure at $500{ }^{\circ} \mathrm{C}$ results in a surface that is smoother at the atomic scale with larger domains measuring about $100 \mathrm{~nm}^{2}$. In addition, this surface contains large single atomic layer vacancy islands, single height steps, and deep pits. The pits have a pyramidal shape, as previously discussed for Fig. 1(b). The vacancy islands have an average length

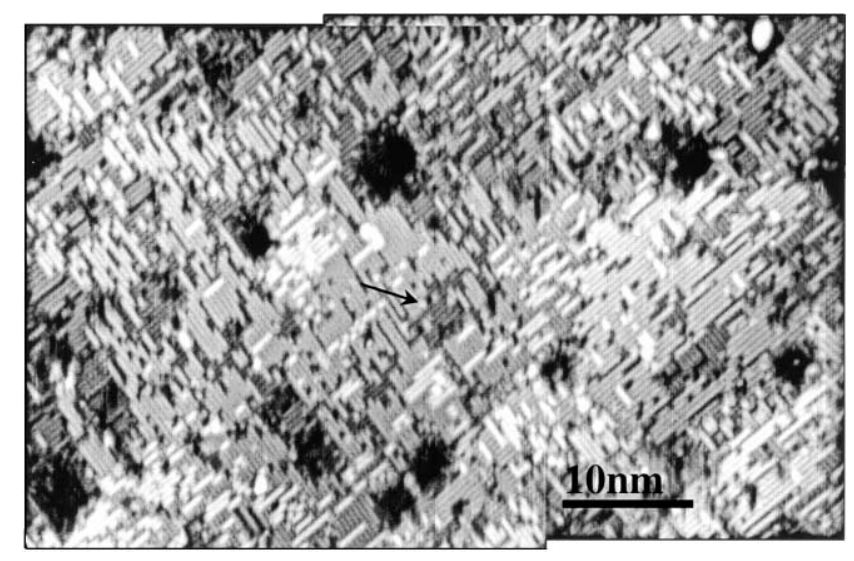

FIG. 3. Nonhydrogen terminated diamond $(100)-(2 \times 1)$ surface after atomic hydrogen exposure for $5 \mathrm{~min}$ at $500{ }^{\circ} \mathrm{C}$. along the dimer rows that is 2.1 times greater than the average length perpendicular to the dimer rows. Both $S_{A}$ and $S_{B}$ steps are rough. These observations show that etching occurs both parallel and perpendicular to the dimer rows. As shown in Figs. 4 and 5, exposure at $1000{ }^{\circ} \mathrm{C}$ results in a smooth surface with the largest domains of the three experiments measuring approximately $350 \mathrm{~nm}^{2}$. Large pits are not observed and vacancy islands consist mainly of single vacancy rows in the direction of the dimer rows. $S_{B}$ steps are rough and $S_{A}$ steps are smooth, showing that etching has a low probability of occurring at $S_{A}$ steps. In addition, double type $A$ steps, denoted $D_{A}$ in Figs. 4 and 5, are observed in which the middle $S_{B}$ step has been completely etched. These observations show that etching at $1000{ }^{\circ} \mathrm{C}$ is highly anisotropic, with the etch rate along the dimer rows much greater than that perpendicular to the rows. In contrast, air STM experiments on epitaxial diamond (100) films that are grown on viscinal diamond (100) substrates and not etched by atomic hydrogen after growth show a smooth surface consisting of double type $B, D_{B}$, steps formed by dimer row extension [19].

The anisotropic etching in the direction of dimer rows supports recent theoretical models of the growth of smooth diamond (100) films. Figure 6 shows a schematic of the diamond (100)- $(2 \times 1): \mathrm{H}$ surface. In theoretical models, growth on this surface occurs by the adsorption of $\mathrm{CH}_{3}$ on surface sites where hydrogen has been abstracted [3]. The adsorbates then form methylene bridges and dimers. In the anisotropic etching model described in Ref. [12], extension of the dimer row at atoms 1 and 2 in Fig. 6 can occur as long as hydrocarbons have not adsorbed at the dimer bonds to their right. For example, the presence of a methylene bridge between atoms 3 and 4 will prevent adsorption of $\mathrm{CH}_{3}$ at atoms 3 or 1 due to steric repulsion, and growth will wait until this methylene bridge is etched. Growth of dimers at $S_{A}$ steps, corresponding to atoms 5 and 6 in Fig. 6, is not kinematically stable against etching because all dimers grown at $S_{A}$ steps are similar to dimers at the

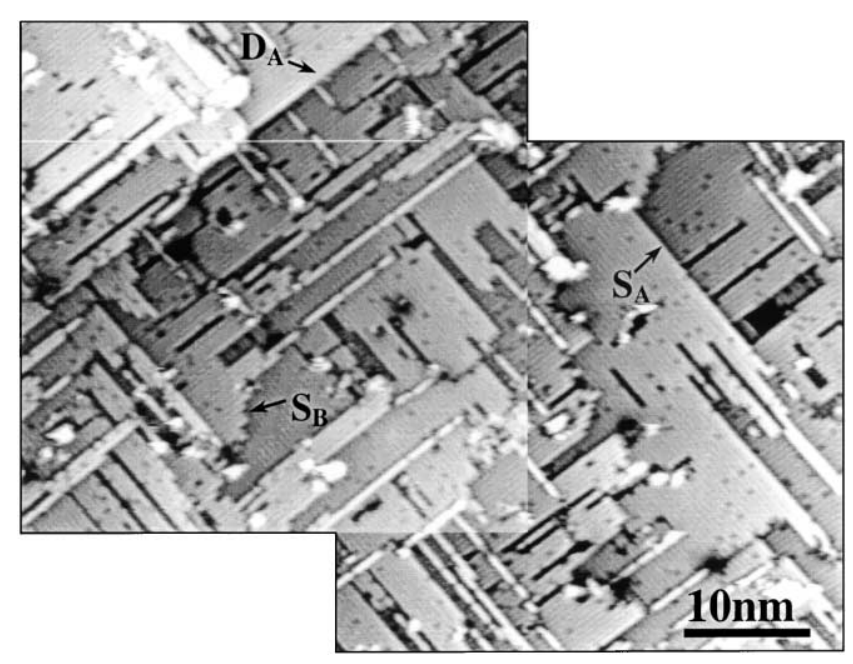

FIG. 4. Nonhydrogen terminated diamond $(100)-(2 \times 1)$ surface after atomic hydrogen exposure for $5 \mathrm{~min}$ at $1000{ }^{\circ} \mathrm{C}$. 


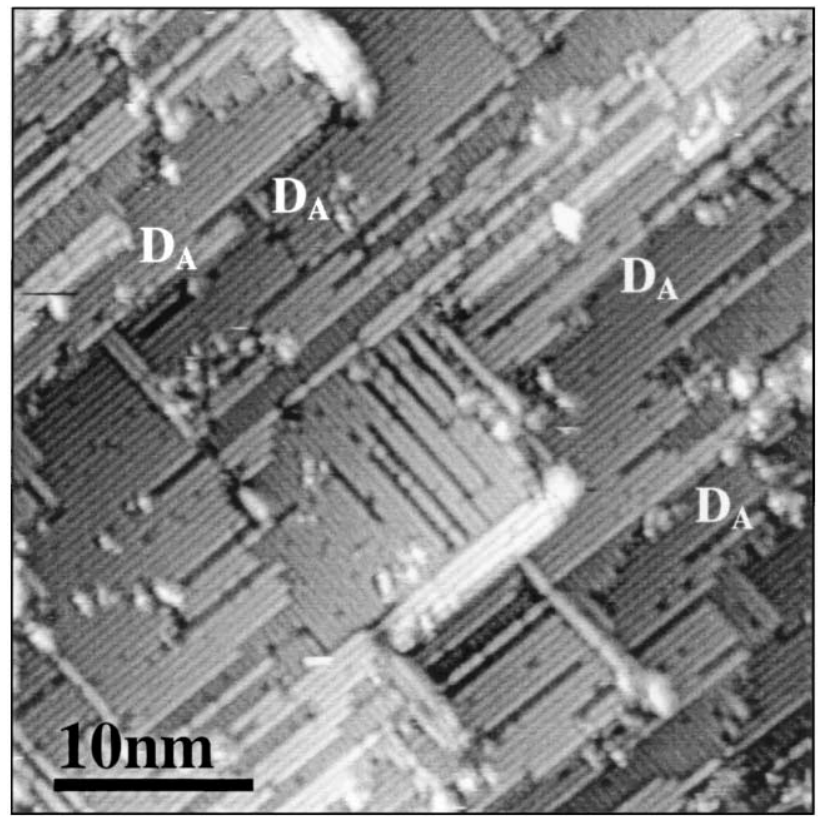

FIG. 5. Nonhydrogen terminated diamond $(100)-(2 \times 1)$ surface after atomic hydrogen exposure for $5 \mathrm{~min}$ at $1000{ }^{\circ} \mathrm{C}$. Observed are various $D_{A}$ steps.

ends of $S_{B}$ steps and can be etched [13]. In contrast, only the last dimer in a row of an $S_{B}$ step has a high probability of being etched. For example, atoms 7 and 8 can be etched only after atoms 9 and 10 are etched. Therefore, growth at $S_{B}$ steps can proceed via dimer row extension and produce a continuous monolayer. Our results provide direct evidence for this model. A recent model that considers the preferential etching of under-coordinated atoms predicts the observed growth rate and a smooth surface [11].

There have been reports of LEED studies of the etching of diamond (100) by atomic hydrogen produced by a microwave plasma [20], and a comparison between etching resulting from atomic hydrogen produced by a hottungsten filament and a microwave plasma by Cheng et al. [21]. In Ref. [21], it was concluded that atomic hydrogen produced by a hot filament made the diamond (100) surface rough with (111) facets, and atomic hydrogen produced by a microwave plasma made the diamond (100) surface smooth. It was suggested that the difference is caused by the higher velocity ions in a hydrogen plasma [21]. Our results explain the observations of Cheng et al. that the hot-tungsten filament technique produced a rough diamond (100) surface. The final step of Cheng et al. was an atomic hydrogen exposure at $T=527^{\circ} \mathrm{C}$ that was intended only to hydrogen terminate the surface. Our results show that exposure of diamond (100) to atomic hydrogen at $T \approx 500{ }^{\circ} \mathrm{C}$ results in a pitted surface with (111) facets.

In summary, we have used UHV STM to study the temperature dependence of the etching of diamond (100) by atomic hydrogen. We find that etching by atomic hydrogen is highly temperature dependent resulting in a rough and pitted surface at $T \approx 200$ and $500{ }^{\circ} \mathrm{C}$, respectively. At $T \approx 1000{ }^{\circ} \mathrm{C}$, etching occurs predominantly in the di-

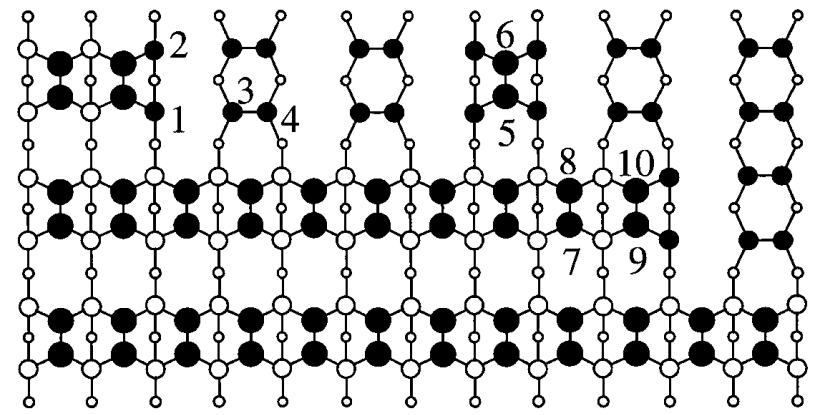

FIG. 6. Schematic of the diamond $(100)-(2 \times 1)$ surface. The large, intermediate, and small circles represent the top, second, and third layer carbon atoms, respectively. The shaded carbon atoms are hydrogen terminated.

rection of dimer rows. This anisotropic etching supports recent theoretical models for the growth of smooth diamond (100) films. Our technique of imaging the nonhydrogen terminated diamond $(100)-(2 \times 1)$ surface using UHV STM can be used to study other adsorbates and processes at the atomic scale. Such studies are crucial in understanding the growth and properties of diamond.

This work was supported by the National Science Foundation under Award No. DMR-0074636, and the Texas Advanced Technology Program under Award No. 0035940048-1999.

[1] B. V. Spitzyn, L. L. Bouilov, and B. V. Deryaguin, J. Cryst. Growth 52, 219 (1981).

[2] Handbook of Industrial Diamonds and Diamond Films, edited by M. Prelas, G. Popovici, and L. Bigelow (Marcel Dekker, New York, 1998).

[3] D. Goodwin and J. Butler, in Handbook of Industrial Diamonds and Diamond Films (Ref. [2]), pp. 527-581.

[4] J. Boland, Surf. Sci. 262, 17 (1992).

[5] Scanning Tunneling Microscopy, edited by J. Stroscio and W. Kaiser (Academic, Boston, 1993).

[6] T. Tsuno et al., Jpn. J. Appl. Phys. 30, 1063 (1991).

[7] H. Busmann and I. Hertel, Carbon 36, 391 (1998).

[8] Y. Kuang et al., Appl. Phys. Lett. 67, 3721 (1995).

[9] T. Tsuno et al., Jpn. J. Appl. Phys. 35, 4724 (1996).

[10] M. Frenklach and S. Skokov, J. Phys. Chem. B 101, 3025 (1997).

[11] C. Battaile et al., J. Chem. Phys. 111, 4291 (1999).

[12] M. Zhu et al., in Proceedings of the Third International Symposium on Diamond Materials, edited by J. Dismukes and K. Ravi (The Electrochemical Society, Pennington, 1993), pp. 138-145.

[13] J. Cui et al., J. Appl. Phys. 83, 7929 (1998).

[14] R. E. Stallcup et al., Ph.D. dissertation, University of North Texas, 2000.

[15] Y. Kim et al., J. Vac. Sci. Technol. B 16, 2079 (1998).

[16] B. Thoms and J. Butler, Surf. Sci. 328, 291 (1995).

[17] X. Jiang and C. Rickers, Appl. Phys. Lett. 75, 3935 (1999).

[18] S. Lee and G. Apai, Phys. Rev. B 48, 2684 (1993).

[19] T. Tsuno et al., Appl. Phys. Lett. 64, 572 (1994).

[20] B. Thoms et al., Appl. Phys. Lett. 65, 2957 (1994).

[21] C. Cheng et al., Phys. Rev. Lett. 78, 3713 (1997). 